\title{
Thrombotic Microangiopathy Secondary to Steroid Pulse Therapy Administered for Refractory Nephrotic Syndrome
}

\author{
Tomotsune Miyamoto, Yozo Ishikawa, Junya Yamamoto, \\ Tsuyoshi Yamamura and Tetsuya Kawata
}

\begin{abstract}
A 79-year-old woman with familial hyperlipidemia was treated with low-density lipoprotein apheresis. She was hospitalized due to fatigue and edema, and massive proteinuria was discovered. Renal biopsy revealed no distinct abnormalities, thus suggesting a diagnosis of minimal change nephrotic syndrome. She developed acute kidney injury and hemodialysis was initiated. Two series of steroid pulse therapy were given, but the proteinuria did not decrease. Thereafter, she developed thrombocytopenia and fell into a stupor. Thrombotic microangiopathy (TMA) was the most likely diagnosis. Plasma exchange was initiated, resulting in improvements in platelet counts and in her level of consciousness. Clinicians should therefore be aware that TMA can occur as a result of steroid pulse therapy.
\end{abstract}

Key words: thrombotic microangiopathy, nephrotic syndrome, steroid

(Intern Med 52: 2099-2103, 2013)

(DOI: 10.2169/internalmedicine.52.0470)

\section{Introduction}

Thrombotic microangiopathy (TMA) represents a spectrum of diseases that can result in microembolisms and organ failure. TMA is considered to be a form of both thrombotic thrombocytopenic purpura (TTP), which manifests in an altered mental status, and hemolytic uremic syndrome (HUS), which manifests in renal failure. TTP may be caused by a disintegrin-like and metalloproteinase with thrombospondin type 1 motifs 13 (ADAMTS13) deficiency, while HUS accompanied by diarrhea can arise from an infection with enterohemorrhagic Escherichia coli (EHEC). Complement component deficiencies can induce atypical HUS without diarrhea (1).

Endothelial injuries of various causes can induce TMA. Plasma exchange (PE) has been established as the treatment of choice for TTP, but its efficacy is controversial when the pathogenesis of the disease does not involve an ADAMTS13 deficiency. We herein report a case of TMA that developed after the initiation of steroid pulse therapy for the management of severe nephrotic syndrome.

\section{Case Report}

A 79-year-old woman complained of bilateral lower extremity edema and fatigue. At 23 years of age, she was noted to have a total cholesterol level of $>600 \mathrm{mg} / \mathrm{dL}$ and was therefore diagnosed with familial hyperlipidemia that was not responsive to statin therapy. Her son also had hyperlipidemia. At 69 years of age, she underwent coronary artery bypass graft surgery due to angina pectoris. Following the operation, she underwent biweekly low-density lipoprotein (LDL) apheresis to address her severe hyperlipidemia. She had neither proteinuria nor occult hematuria until four months prior to her current presentation, when she developed proteinuria. Two weeks prior to her current presentation, she developed edema and weight gain. She was hospitalized at another facility because of fatigue and edema, where she was noted to have massive proteinuria. At that time, she was transferred to our hospital for evaluation and management of her nephrotic syndrome.

Urinalysis showed 4+ proteinuria and a protein-creatinine ratio of $15.7 \mathrm{~g} / \mathrm{gCr}$ (24-hour proteinuria showed a protein excretion of $10.8 \mathrm{~g}$ ). The laboratory examination revealed a

Department of Nephrology, Hokkaido Medical Center, Japan

Received for publication February 26, 2013; Accepted for publication May 7, 2013

Correspondence to Dr. Tomotsune Miyamoto, tmiyamo@hok-mc.hosp.go.jp 


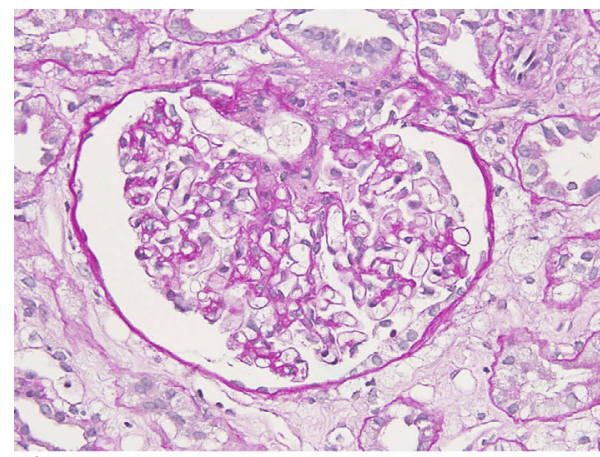

A

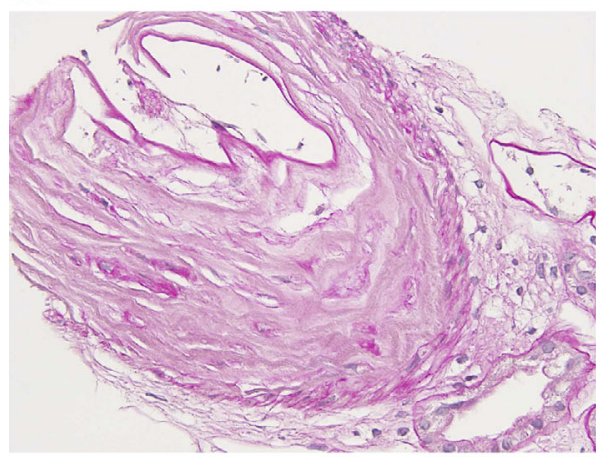

$\mathrm{C}$

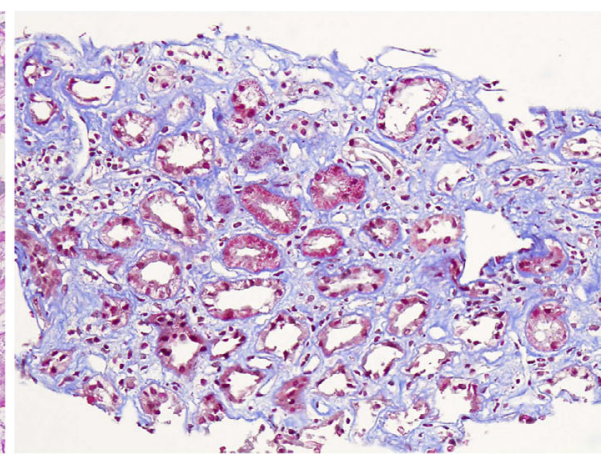

B

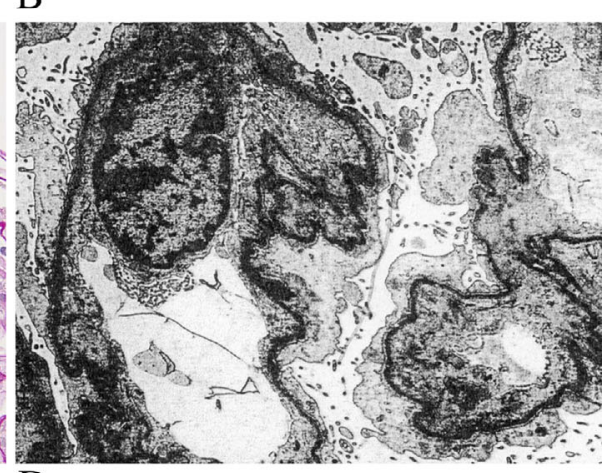

$\mathrm{D}$

Figure 1. Renal biopsy findings. A) Periodic acid Schiff stain, $\times 400$. A glomerulus is normal. Mesangium matrix enlargement and proliferation of mesangium cells are not obvious. B) Masson stain, $\times 200$. About $20 \%$ of interstitium show fibrosis, which indicates ischemic features. C) Periodic acid Schiff stain, $\times 400$. An interlobular artery shows laminar intimal thickening and severe narrowing. D) Electron microscopy, $\times 5,000$. Marked effacement of the podocyte foot processes is shown. Endothelial changes and electron-dense deposits are not detected.

urea nitrogen level of $21.5 \mathrm{mg} / \mathrm{dL}$ and a creatinine $(\mathrm{Cr})$ level of $0.76 \mathrm{mg} / \mathrm{dL}$. Her liver function was within normal limits, but her serum total protein was low at $3.9 \mathrm{~g} / \mathrm{dL}$ and her serum albumin was $1.8 \mathrm{~g} / \mathrm{dL}$. On the day after an LDL apheresis treatment, her LDL cholesterol was $154 \mathrm{mg} / \mathrm{dL}$ and her triglycerides were $270 \mathrm{mg} / \mathrm{dL}$. Her lipid profile was consistent with type IIb hyperlipidemia. Antinuclear antibody testing was negative and the complement levels were within normal limits. Computed tomography showed pleural effusion and ascites.

Heparin was continuously infused to prevent thrombosis due to an excessive hemoconcentration. Diuretic therapy was administered, and resulted in a resolution of the edema, pleural effusions and ascites. LDL apheresis was continued every 2 weeks. Renal biopsy was performed, and the patient was discharged from the hospital to await the results of renal pathology.

Three weeks later, she was rehospitalized due to severe edema. At this point, urinalysis showed a protein-creatinine ratio of $19.6 \mathrm{~g} / \mathrm{gCr}$ and a serum albumin level of $1.1 \mathrm{~g} / \mathrm{dL}$. Heparin was reinstituted to prevent thrombosis. Renal biopsy showed no definite abnormalities in the glomeruli (Fig. 1), even though approximately $20 \%$ of the interstitium showed fibrosis. The interlobular arteries had laminar intimal thickening and narrowing due to arteriosclerosis. The immunofluorescence testing did not show a specific pattern.
Electron microscopy showed podocyte foot process effacement without any endothelial changes. These observations suggested a diagnosis of minimal change nephrotic syndrome (MCNS). Hence, steroid pulse therapy (methylprednisolone at a dosage of $500 \mathrm{mg}$ daily for 3 days) was administered. Despite this treatment, proteinuria did not decrease and the patient subsequently developed acute kidney injury and oliguria. Thus, hemodialysis was initiated. A second course of steroid pulse therapy was administered, followed by chronic therapy with prednisolone (30 $\mathrm{mg}$ daily). However, proteinuria, pleural effusion and ascites persisted. Hemodialysis was difficult to perform due to the deterioration of the patient's general condition.

Immediately after the second course of steroid pulse therapy, the patient complained of numbness in both upper limbs. Brain magnetic resonance imaging showed a small area of high intensity in the left parietal lobe in diffusionweighted images, which led to the suspicion of a minor acute infarction (Fig. 2). Her platelet count decreased from $214,000 / \mu \mathrm{L}$ to $169,000 / \mu \mathrm{L}$ over a 2 -day period. Three days later, the patient vomited with scant hematemesis. Gastroendoscopy showed blood oozing from the gastric mucosa in the context of a platelet count of $19,000 / \mu \mathrm{L}$. Eight days later, her platelet count was $6,000 / \mu \mathrm{L}$ (Fig. 3). She fell into a stupor and began to experience clonic spasms and purpura of both lower extremities. Her hemoglobin level decreased 


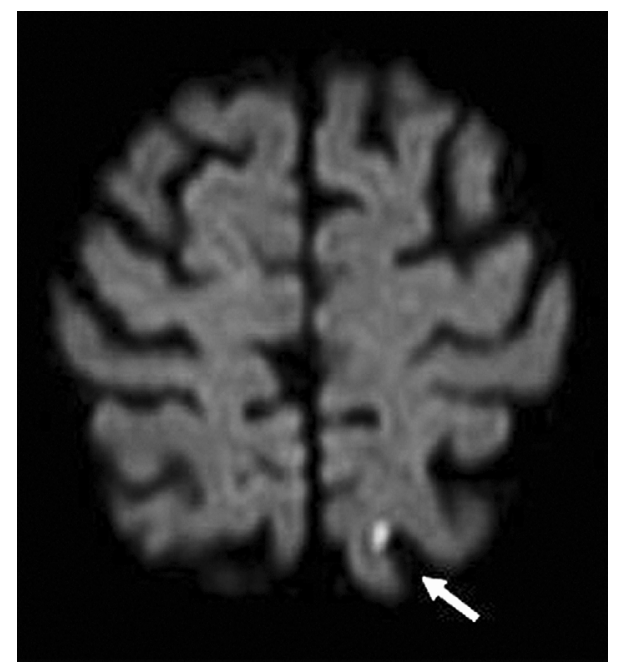

Figure 2. Brain magnetic resonance imaging. There is a small area of high intensity in the left parietal lobe in diffusion-weighted image, which leads to the suspicion of minor acute infarction (arrow).

from $14.1 \mathrm{~g} / \mathrm{dL}$ to $11.4 \mathrm{~g} / \mathrm{dL}$ over a 2-day period, while her haptoglobin level decreased to $2 \mathrm{mg} / \mathrm{dL}$ and her lactate dehydrogenase level increased to 1,590 U/L. These findings were suggestive of hemolytic anemia with upper gastrointestinal bleeding.

Since LDL apheresis had been performed for the previous 10 years with heparin, which had been continuously infused at her first admission without a decrease in platelets, heparin-induced thrombocytopenia was ruled out. The laboratory coagulation data showed normal fibrinogen levels of $216 \mathrm{mg} / \mathrm{dL}$ and only slightly elevated levels of prothrombin time-international normalized ratio (PT-INR) (1.45) and Ddimer $(8.3 \mu \mathrm{g} / \mathrm{mL})$. Her C-reactive protein level was 1.45 $\mathrm{mg} / \mathrm{dL}$ and her body temperature was normal, both of which suggested that she did not have an active infection. We therefore ruled out disseminated intravascular coagulation due to sepsis. Her symptoms and renal pathology were not consistent with systemic lupus erythematosus (SLE). Although the antibodies directed against cardiolipin were not evaluated, antiphospholipid antibody syndrome in SLE was also not supported. Although fragmented red blood cells were not detected, the combination of hemolytic anemia and thrombocytopenia with various ischemic symptoms clinically suggested the presence of TMA.

PE treatment was immediately initiated without waiting for the result of the ADAMTS13 activity. PE using 30 units of fresh frozen plasma was performed with continuous hemodiafiltration over the first 2 days. Subsequently, PE with hemodialysis was performed intermittently. Steroid therapy was discontinued, as the treatment was not positively affecting the nephrotic syndrome. PE was performed eight times in total until her platelet counts recovered. Her ADAMTS13 activity was subsequently reported as $39.6 \%$. She had become anuric after the diagnosis of TMA, but her renal function did recover. Hemodialysis was terminated.
The edema resolved and proteinuria decreased to $5.5 \mathrm{~g} / \mathrm{gCr}$. Thereafter, her proteinuria increased slowly, but her Cr levels remained at $1.0 \mathrm{mg} / \mathrm{dL}$ without hemodialysis. She has now been symptom-free for over 1 year.

\section{Discussion}

This report describes the case of a patient who developed TMA after the initiation of steroid pulse therapy administered for the management of refractory nephrotic syndrome. This case did not strictly satisfy the criteria for TTP as an ADMATS13 inhibitor was not detected. Indeed, the ADAMTS13 activity in the present case was 39.6\%, whereas TTP generally develops at an ADAMTS13 activity of $\leq 5 \%$. Further, the lack of diarrhea or signs of infection argue against enterohemorrhagic E. coli-hemolytic uremic syndrome (EHEC-HUS), and the autoantibodies to the complement component are unlikely to appear in the context of steroid therapy, making atypical HUS an unlikely diagnosis. Steroids are used to inhibit the generation of ADAMTS13 antibodies in patients with TTP, but steroids may also cause TMA. Steroid therapy is a risk factor for the development of scleroderma renal crisis (2), the pathology of which is consistent with that of TMA. Furthermore, cases of TMA developing after steroid therapy in a patient with microscopic polyangiitis (3) and TMA secondary to steroid pulse therapy for graft-versus-host disease (polyserositis and membranous nephropathy) (4) have been reported.

In the present case, LDL apheresis was utilized for 10 years to address familial hyperlipidemia. Chronic hyperlipidemia can induce endothelial dysfunction and arteriosclerosis (5). Hence, severe arteriosclerosis and interstitial fibrosis, which also resulted in the patient's coronary ischemia, were likely involved in her renal pathology. Severe nephrotic syndrome leading to an acute kidney injury can induce hypercoagulation with the risk of venous and arterial thromboembolic events (6). Under such circumstances, TMA can be evoked due to free radical production, hypercoagulation and endothelial injury (7). The endothelial injury further facilitates coagulation in nephrotic syndrome (8), giving rise to a vicious cycle that continually worsens the TMA. However, the pathogenic mechanisms in our patient were not well understood. Renal biopsy, which was performed at her first admission for nephrotic syndrome, when she had not yet developed clinical TMA, did not show thrombosis under light microscopy or endothelial injury under electron microscopy.

The onset of thrombosis in our patient seems to have occurred immediately after the second steroid pulse therapy. At that point, she complained of numbness and her platelet count began to decrease, thus suggesting that TMA developed as an acute thrombotic event due to steroid therapy.

Corticosteroids can also promote thrombosis by suppressing prostacyclin, which normally inhibits platelet aggregation (9). Although the suppression of prostacyclin alone is not sufficient to cause TMA, its occurrence at the same time as hypercoagulability due to severe nephrotic syndrome can 


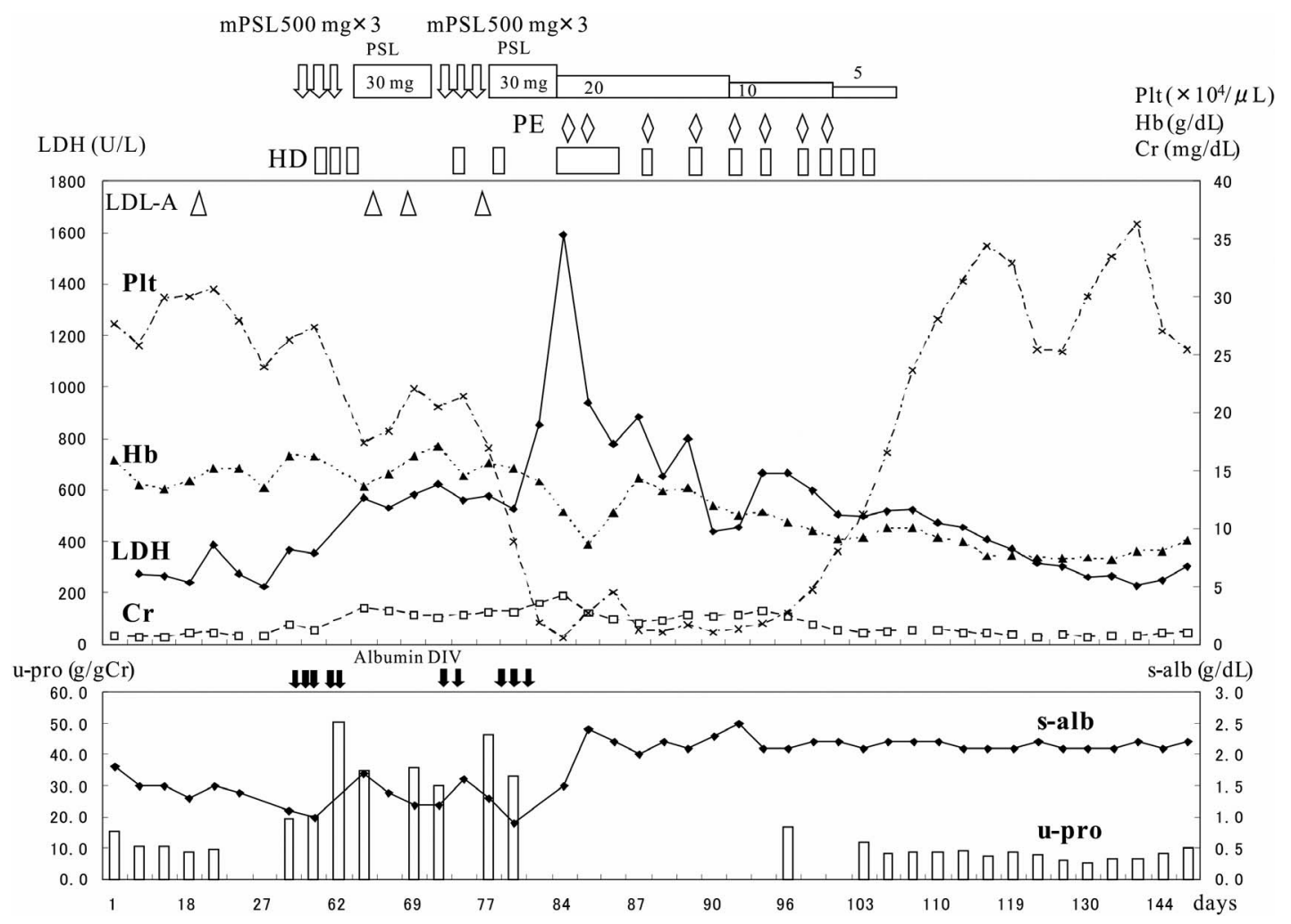

Figure 3. Clinical course. Cr: creatinine, Hb: hemoglobin, HD: hemodialysis, LDH: lactate dehydrogenase, LDL-A: low-density lipoprotein apheresis, mPSL: methylprednisolone, PE: plasma exchange, PIt: platelet count, PSL: prednisolone, s-alb: serum albumin, u-pro: proteinuria

lead to a collapse of the platelet balance, leading to TMA. It is also possible that steroids could induce endothelial dysfunction (10), which enhances platelet aggregation.

Hypertension due to steroids can induce TMA (3). However, the average blood pressure in the present case was $108 / 61 \mathrm{mmHg}$ before steroid therapy and 114/70 $\mathrm{mmHg}$ post steroid therapy. Therefore, accelerated hypertension appears to be irrelevant in this case.

Ordinarily, steroid pulse therapy should result in an improvement of nephrotic syndrome, suggesting that perhaps the underlying disease was focal segmental glomerulosclerosis rather than MCNS. Repeated steroid pulse therapy in patients with steroid-resistant nephrotic syndrome with risk factors such as familial hyperlipidemia could provoke TMA. A specific risk factor in the present case was familial hyperlipidemia, which required 10 years of LDL apheresis therapy. Severe arteriosclerosis and possible endothelial dysfunction might have contributed to the TMA. A poor response to steroid pulse therapy and the magnitude of proteinuria and serum albumin levels might have been other risk factors, all of which contributed to the development of secondary TMA in our patient.

Transplantation-associated TMA is a form of TMA for which plasma exchange is neither effective nor recommended (11). Malignant hypertension also induces TMA due to endothelial injury, but, again, $\mathrm{PE}$ is not necessary as the condition resolves once blood pressure is controlled. Therefore, clinicians should be aware of the fact that not all cases of TMA require PE. In the face of the life-threatening symptoms in our patient, PE was immediately administered, even before the results of the ADAMTS13 inhibitor testing were available. The therapy resulted in a resolution of TMA, a reduction of proteinuria and the restoration of renal function. Based on these improvements in the nephrotic syndrome, we were able to terminate the hemodialysis treatments. PE was not thought to have directly cured the TMA, because ADAMTS13 deficiency was not present. It is not clear whether simply discontinuing the steroids could have helped to resolve TMA, even though decreasing steroids is essential, but not sufficient, to re-balance platelet coagulation once steroid-induced TMA develops. PE can ameliorate nephrotic syndrome (12), which can otherwise cause platelet coagulation. As a result, the alleviation of nephrotic syndrome is likely to normalize the platelet activity and result in a resolution of TMA.

In conclusion, steroids can induce secondary TMA in the setting of a hypercoagulable state due to severe nephrotic syndrome, likely via their effects on platelet aggregation. This type of TMA does not involve an ADAMTS13 deficiency. However, the detailed pathological mechanism, including endothelial injury, is unknown. Further studies are needed to fully elucidate this phenomenon and to determine what risk factors contribute to the development of TMA secondary to steroid pulse therapy.

The authors state that they have no Conflict of Interest (COI). 


\section{References}

1. Besbas N, Karpman D, Landau D, et al. A classification of hemolytic uremic syndrome and thrombotic thrombocytopenic purpura and related disorders. Kidney Int 70: 423-431, 2006.

2. Denton CP, Lapadula G, Mounthon L, Müller-Ladner U. Renal complications and scleroderma renal crisis. Rheumatology 48: iii32-iii35, 2009.

3. Yoshioka K, Hattori T, Isaka Y, et al. Thrombotic microangiopathy due to malignant hypertension following corticosteroid therapy for microscopic polyangiitis. Intern Med 46: 785-788, 2007.

4. Ishikawa Y, Nishio S, Sasaki H, et al. Transplantation-associated thrombotic microangiopathy after steroid pulse therapy for polyserositis related to graft-versus-host disease. Clin Exp Nephrol 15: 179-183, 2011.

5. Raman KG, Gandley RE, Rohland J, et al. Early hypercholesterolemia contributes to vasomotor dysfunction and injury associated atherogenesis that can be inhibited by nitric oxide. J Vasc Surg 53: 754-763, 2011.

6. Mahmoodi BK, ten Kate MK, Waanders F, et al. High absolute risks and predictors of venous and arterial thoromboembolic events in patients with nephrotic syndrome. Circulation 117: 224-
$230,2008$.

7. Morita S, Sakai T, Okamoto N, et al. Hemolytic uremic syndrome associated with immunoglobulin A nephropathy: a case report and review of cases of hemolytic uremic syndrome with glomerular disease. Intern Med 38: 495-499, 1999.

8. Malyszko J, Malyszko JS, Mysliwiec M. Markers of endothelial cell injury and thrombin activatable fibrinolysis inhibitor in nephrotic syndrome. Blood Coagul Fibrinolysis 13: 615-621, 2002.

9. Lewis GD, Campbell WB, Johnson AR. Inhibition of prostaglandin synthesis by glucocorticoids in human endothelial cells. Endocrinology 119: 62-69, 1986.

10. Balkaya M, Prinz V, Custodis F, et al. Stress worsens endothelial function and ischemic stroke via glucocorticoids. Stroke 42: 32583264, 2011.

11. Scully M, Hunt BJ, Benjamin S, et al. British Committee for Standards in Haematology. Guidelines on the diagnosis and management of thrombotic thrombocytopenic purpura and other thrombotic microangiopathies. Br J Haematol 158: 323-335, 2012.

12. Yokoyama H, Wada T, Zhang W, Yamaya H, Asaka M. Advances in apheresis therapy for glomerular disease. Clin Exp Nephrol 11: 122-127, 2007.

(C) 2013 The Japanese Society of Internal Medicine http://www.naika.or.jp/imonline/index.html 